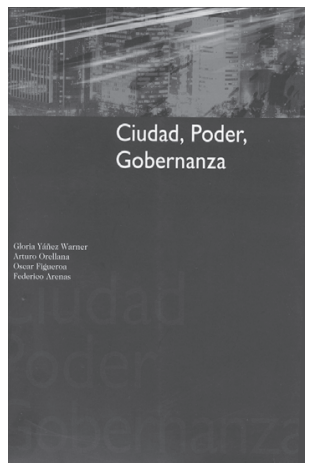

\title{
Gloria Yáñez Warner, Arturo Orellana, Óscar Figueroa, Federico Arenas (Editores). Ciudad, Poder, Gobernanza
}

\author{
Santiago de Chile: Instituto de Estudios Urbanos, U. \\ Católica de Chile, Serie EURE Libros - Instituto de \\ Geografía, U. Católica de Chile, Serie GEOlibros No 9 - \\ Colección Rideal, 2008, 455 p.
}

\author{
Andrea Vásquez ${ }^{1}$
}

La gestión de las ciudades es un tema de debate permanente, sobre todo cuando la escala de la ciudad se exacerba a la escala metropolitana, aumentando consigo, la superficie ocupada y la población que reside en ellas.

Ante esta problemática emerge el concepto de gobernanza, entendiéndose este, en palabras de Boisier (2006) como la forma de gobernar que tiene el gobierno, en ese sentido, corresponde a una forma de gobernabilidad, definida esta última como la capacidad política que tiene el gobierno para gobernar, incluyendo en ese gobierno, por definición, la diversidad de intereses presentes en él, ya sea desde las distintas posturas que tengan los agentes directos que constituyen al gobierno (en su sentido común), como a los ciudadanos, los cuales idealmente deberían manifestar sus intereses en forma autorregulada (como sociedad) a decir de Mayntz (2001). En suma, la participación ciudadana en forma activa, es un componente fundamental de la gobernanza y permite, por la misma razón, la constitución de un buen gobierno.

Sumado a lo anterior, Mayntz, reconocido por su aporte a la temática en cuestión, aduce que a la sociedad subyace una serie de intereses personales y relaciones de po-

\footnotetext{
1 Estudiante de pregrado, Instituto de Geografía, Pontificia Universidad Católica de Chile (Chile). Email: arvasque@uc.cl
}

der, los cuales pueden ser consensuados, si es que esa sociedad tiene la capacidad de autorregularse, confiando en que ese consenso de intereses, será representado en la gobernabilidad, a través de la gobernanza. No obstante, lo anterior, si bien suma antecedentes y complementa la definición expresada por Boisier, la eleva a un nivel que bien se podría remontar al modelo de estado de Hobbes con el Leviatán. La pregunta que surge, por tanto, ¿Existe esa representatividad? La democracia, con la representatividad de la mayoría, si bien no se condice exactamente con la descripción realizada por Mayntz, al menos se acerca a una forma de regular la sociedad civil y representarla.

A su vez, Mayntz distingue entre el concepto de gobernanza y gobernanza moderna, definiendo esta última como una forma cooperativa de gobernar, es decir, sin que los actores involucrados (públicos, privados, ciudadanos) se relacionen en base a una jerarquía. Esto tampoco implica el caos, simplemente, que si lo anterior está regulado por una sociedad civil entendida como "una comunidad de ciudadanos, de individuos que disfrutan de igualdad ante la ley, así como de un conjunto de derechos fundamentales, y cuya libertad de perseguir sus metas privadas solo está restringida por el mismo derecho de los demás, y por los derechos de la res publica que derivan de su condición de ciudadanos" (Mayntz, 2001: 3), cuyo gobierno los representa de forma efectiva, entonces es posible la gobernanza moderna. Todo esto incluye el concepto de 
poder, porque los actores podrán intervenir en la medida que ejerzan más o menos poder. Pues bien, en una gobernanza moderna, ese poder no está concentrado en forma hegemónica en los actores públicos, ni privados, ni en los ciudadanos, sino que se encuentra disperso entre ellos. Si se consideran los tres actores, el poder estaría tripartito.

A lo anterior se suma una tercera posición, descrita por Parrado (2005), quien, en su intento por medir el estado de la gobernanza, concretiza la definición de la misma en que los distintos actores interactúan (sin hacer referencia al poder), para influir positivamente en la calidad de vida de los habitantes de un área determinada. En vista de lo anterior, surge la interrogante de si dependiendo de la escala, ¿se puede hablar de gobernanza? Y, si la problemática se sitúa a escala metropolitana, ¿se pueden aunar intereses contrapuestos? ¿Cómo se autorregulan esas sociedades, cómo se logra una participación efectiva?

Los párrafos precedentes sitúan la gobernanza como un concepto abstracto, difícil de aterrizar a un ejemplo concreto. No obstante, en "Ciudad, poder, gobernanza", el concepto da pie no solo a reflexiones acerca de los conceptos involucrados, sino también a aplicaciones concretas del mismo.

El libro consta de cuatro capítulos. El primero de ellos se titula "La dimensión espacial del poder". Introduce al lector con una breve reflexión acerca de los conceptos de gobernabilidad, gobernanza y poder, sumado al concepto de escala. Posteriormente, pese a que se ubican en el mismo capítulo, se presentan artículos mixtos, es decir, integran la reflexión con la aplicación. Un ejemplo de ello es "Obstáculos para la gobernanza de los espacios metropolitanos chilenos: fragmentación institucional, desarticulación instrumental, atomización social". Finalmente, el primer capítulo termina con tres ejemplos, cuestionando las reflexiones con las que se inicia el texto. Los ejemplos consideran tres áreas distintas: el caso francés, el área metropolitana de Buenos Aires y el área metropolitana de Santiago. Resulta interesante, puesto que permite la comparación de la gobernabilidad en áreas metropolitanas insertas en contextos espaciales diferentes: Europa y América Latina (Sudamérica).

El segundo capítulo se titula "Periferia, intersticios y redes metropolitanas". A diferencia del primer capítulo, el segundo contiene una mayor diversidad de temas. De los cinco artículos que contiene, tres están referidos al caso chileno (referido específicamente al área metropolitana de Santiago y sus alrededores), desde distintas ópticas, dos de ellos se aproximan a la realidad rural y rururbana, respectivamente, desde los organismos de gestión para dichas áreas. El tercero, realiza una reflexión basada en la historia de la vivienda social en el Área Metropolitana de Santiago. El capítulo finaliza con un ejemplo aplicado al transporte en Europa. Este capítulo permite no solo comparar dos áreas distintas, pero que conviven a cortas distancias, como son los espacios rurubanos y rurales versus el área metropolitana de Santiago, porque, evidentemente, la gestión con que se administra el territorio (sobre todo el urbano) tendrá incidencia en los espacios aledaños a este. También permite comparar el caso chileno con el europeo, con la gobernanza aplicada al transporte, en el caso de Europa. Así, se distinguen diferentes escalas espaciales, pero también distintos niveles de gobernanza y gobernabilidad.

El tercer capítulo, titulado "Proyectos y actores urbanos", así como el segundo capítulo, contiene temáticas variadas. No obstante, es importante resaltar la diversidad de enfoques que se presentan en este capítulo, no solo por la perspectiva desde la que narran los autores, sino por la aplicabilidad de los conceptos, resumidos en ciudad, poder y gobernanza, a escalas no solo espaciales, sino que temáticamente distintas. Tales como lo retratan los artículos referidos a la ley de barrios de Cataluña, los proyectos urbanos conflictivos en Munich, Alemania, la representación parlamentaria en las áreas metropolitanas chilenas, el caso de la parte norte de los Pirineos del sur y el mercado de trabajo de mujeres asociado al buen gobierno.

Por último, el cuarto capítulo, titulado "Sustentabilidad urbana y territorial", finali- 
za con tres artículos, dos de los cuales describen la sustentabilidad ambiental aplicada al caso de metrópolis chilenas (Santiago, Valparaíso y Concepción), enfatizando el desarrollo de las islas de calor urbano en las metrópolis, junto con el último, que describe la problemática de la regulación de las aguas para el caso de la región metropolitana de Sâo Paulo. Es importante destacar que el segundo artículo de este capítulo (en el orden descrito por el libro) enlaza el concepto de la gobernanza con la sustentabilidad urbana, dando paso a la reflexión acerca de la aplicación a casos concretos, de los conceptos que inicialmente se situaron en una esfera abstracta.

Así es como, en términos generales, el libro desarrolla reflexiones acerca de los conceptos ya descritos, sumado a la aplicación de los mismos y, uno de los aspectos centrales de la obra: la espacialización y la escala (espacio-temporal) de los ejemplos utilizados.

"Ciudad, poder y gobernanza" es una obra completa, puesto que no deambula en los conceptos, ya que no es la finalidad determinar el estado del arte del debate. Como se expuso en los primeros párrafos, la gestión de las áreas metropolitanas (sobre todo) es un debate actual, pero, como tema, ha sido tratado desde que aparecieron las grandes ciudades. Es difícil conceptualizar y sobre todo, aunar el concepto de gobernabilidad, gobernanza, cuando, luego de la lectura, sobresalen casos dispares. Gobernabilidad como en el caso francés, disímil de la del área metropolitana de Santiago y distante del conflicto que se presenta en Munich, Alemania, por la relocalización del estadio olímpico. Luego de las variadas opiniones acerca del tema, surgen muchas preguntas, sobre todo un aspecto que algunos autores recalcaron más que otros, el contexto en el que se inserta esta gestión. Algunos aducen que en un contexto de globalización prima aún más la escala local, mientras, críticas bien argumentadas aducen que una gestión vertical (léase comuna-provinciaregión-país) no aplica la gobernanza. Porque, es cierto, se puede aplicar la gobernanza a distintos niveles, sobre todo cuando existe democracia, luego, el problema de la representatividad, el problema de la con- fianza en que el gobierno de verdad representa a la sociedad civil. La pregunta que surge, inevitablemente es ¿qué está faltando para la gobernanza? ¿En realidad es un fallo generalizado de los sistemas de gestión, de la autorregulación de la sociedad, de la gubernamentalidad que cita Barton? ¿O será que en un contexto globalizado, priman las particularidades territoriales? Se vuelve a la pregunta de qué fue primero, si el huevo o la gallina. ¿Por qué funciona en países como Alemania y no en países como Francia... o en Chile? Lo claro es que, independiente de las reflexiones que se puedan hacer, existe la inquietud por diagnosticar qué está sucediendo en distintas partes del globo acerca de la manera de hacer gobierno, de ahí, las formas que se empleen para hacer que una sociedad participe activamente, reorganizando los instrumentos de gestión existentes o creando algunos nuevos, eso sí responde a como esté constituida la sociedad civil, no tanto como ausencia o participación, pero sí, de todas maneras, como un actor, como un agente en la forma de hacer ciudad. En ese sentido, la gobernanza no es solo responsabilidad de la máxima jerarquía, sino de cada uno de los constituyentes, ya del país, ya de la región, de la provincia, de la comuna. Esa agencia no es que no se ejerza, sino que se está ejerciendo de una determinada manera... y recogiendo las palabras de Mardones ¡Ya basta de que la ciudad esté en manos de los ingenieros en transporte!

Finalmente, "Ciudad, poder, gobernanza" es un aporte significativo, no solo para la geografía, sino para distintos ámbitos del conocimiento, puesto que presta diversidad de enfoques, diversidad de temáticas y de escalas espaciales. Al mostrar distintos ejemplos del globo, permite no solo acercarse desde la gestión a las distintas ciudades (tanto europeas como latinoamericanas), sino adentrarse más en cómo se está haciendo ciudad en la actualidad, en Chile y en Europa. Es un libro de lectura amena, la diversidad de temáticas abordadas permite ir desde la reflexión a la ejemplificación. Por lo tanto, es recomendable no solo para académicos y estudiantes insertos en la materia, sino para quienes quieran tener un primer acercamiento a los conceptos y a distintos espacios del globo. 


\section{Referencias bibliográficas}

BOISIER, S. Algunas reflexiones para aproximarse al concepto de ciudad-región. Revista Estudios Sociales, 2006, Vol. XIV, № 28, p. 163-190.
MAYNTZ, R. El estado y la sociedad civil en la gobernanza moderna. Revista del CLAD Reforma y Democracia, 2001, № 21, p. 1-8.

PARRADO, S.; LÖFFLER, E.; BOVAIRD, T. Evaluación de la calidad de la gobernanza local: algunas lecciones de la experiencia europea. Revista del CLAD Reforma y Democracia, 2005, № 33, p. 1-15. 